01

\title{
Динамика ионов в атмосферном источнике с фотоионизацией излучением лазерной плазмы
}

\author{
(c) Р.С. Аблизен, ${ }^{1}$ М.А. Монастырский, ${ }^{2}$ М.Г. Скоблин, ${ }^{2}$ А.В. Пенто, ${ }^{1}$ А.Б. Бухарина, ${ }^{1}$ С.М. Никифоров, ${ }^{1}$ \\ A.A. Макаров ${ }^{3}$ \\ ${ }^{1}$ Институт общей физики им. А.М. Прохорова РАН, \\ 119991 Москва, Россия \\ ${ }^{2}$ Thermo Fisher Scientific (Москва), \\ 117485 Москва, Россия \\ ${ }^{3}$ Thermo Fisher Scientific, \\ 28199 Bremen, Germany \\ e-mail: romb05958@gmail.com
}

Поступило в Редакцию 24 декабря 2019 г.

В окончательной редакции 27 мая 2020 г.

Принято к публикации 19 июня 2020 г.

\begin{abstract}
Представлены результаты математического моделирования импульсного источника ионов при атмосферном давлении с ионизацией анализируемого вещества УФ излучением лазерной плазмы. Расчеты проводились на основе разработанной авторами математической модели, описывающей эволюцию ансамбля ионов под действием скрещенных газодинамических и электрических полей с учетом эффектов кулоновского взаимодействия. Модель включает в себя систему уравнений Навье-Стокса для описания формирования газодинамических течений в исследуемом объеме, уравнение Пуассона для определения результирующего распределения электрического поля в этом объеме и уравнение Лоренца для построения ионных траекторий ионов от места их рождения до собирающей диафрагмы. Показана сходимость метода релаксации при численном решении основных уравнений модели. Исследована зависимость ионного тока от приложенного напряжения и показано, что учет конечности времени жизни ионов за счет эффекта рекомбинации позволяет получить хорошее согласие между расчетными и экспериментальными данными.
\end{abstract}

Ключевые слова: лазерная плазма, математическое моделирование, ионный источник, газодинамические и электростатические поля, масс-спектрометрия.

DOI: $10.21883 /$ JTF.2020.12.50113.425-19

\section{Введение}

Методы масс-спектрометрии с ионизацией при атмосферном давлении получили широкое применение в различных областях науки, медицины, фармакологии. В настоящее время наиболее популярными методами ионизации при атмосферном давлении являются методы, использующие непрерывный поток ионов, генерируемых различными видами электрического разряда. К таким методам относятся электроспрей [1], химическая ионизация при атмосферном давлении [2], DART [3], различные виды источников с холодной плазмой [4]. Непрерывный поток ионов создает также фотоионизация с использованием УФ ламп [5]. Существуют также импульсные ионные источники, к которым относится источник с ионизацией органических соединений жестким УФ излучением лазерной плазмы [6] и ионизацией лазерным излучением при атмосферном давлении [7].

Метод ионизации излучением лазерной плазмы, используемый в настоящей работе, обладает рядом особенностей. Лазерная плазма, созданная под воздействием лазерного излучения на поверхности металлической мишени, представляет собой пароплазменное облако продуктов абляции и служит источником мощного УФ излучения. Жесткое широкополосное излучение лазер- ной плазмы способно ионизовать любые соединения и элементы вплоть до благородных газов, при этом в отличие от УФ лампы спектральный диапазон плазменного источника не ограничен оптическими окнами. Другим преимуществом плазменного источника является его способность работать в широком диапазоне газовых сред и давлений.

Различные механизмы и модели лазерной абляции металлов при воздействии субнаносекундных, пикосекундных и фемтосекундных лазерных импульсов рассматривались в ряде работ (см., например, [8-11]). Данные о разнообразных экспериментах по лазерной абляции имеются в работах [12-14].

В частности, в работе [12] с оловом в качестве материала мишени изучены спектральные свойства УФ излучения в вакууме в диапазоне 10-20 nm при плотности мощности падающего лазерного излучения $W=2 \cdot 10^{11}-10^{12} \mathrm{~W} / \mathrm{cm}^{2}$ с длиной волны $\lambda=1.06 \mu \mathrm{m}$ и длительностью лазерного импульса $10 \mathrm{~ns}$. В работе [13] исследованы электронная плотность и температура лазерной плазмы на кремниевой мишени при атмосферном давлении с параметрами падающего излучения $\lambda=284-293 \mathrm{~nm}, W=2 \cdot 10^{10}-8 \cdot 10^{11} \mathrm{~W} / \mathrm{cm}^{2}$ и 3 ns. В работе [14] при $W=5.9 \cdot 10^{9} \mathrm{~W} / \mathrm{cm}^{2}$ и $\Delta T=6 \mathrm{~ns}$ в зависимости от внешнего давления исследованы дина- 
мика расширения плазменного облака и спектральные свойства излучения плазмы, образованной на медной мишени, в диапазоне $\lambda=420-540 \mathrm{~nm}$.

В настоящей работе используются данные экспериментов, выполненных в Институте общей физики им. А.М. Прохорова РАН, по исследованию свойств лазерной плазмы, получаемой в результате абляции мишеней из нержавеющей стали при воздействии субнаносекундных лазерных импульсов.

В масс-спектрометрах рассматриваемого типа ионы, образованные в результате ионизации вещества излучением лазерной плазмы, движутся внутри ионизационной камеры при давлении, близком к атмосферному, до момента их попадания в высоковакуумную часть массспектрометра. Движение ионов на данном этапе определяется динамикой газовых потоков и распределением создаваемого электродами электрического поля внутри камеры. Время, в течение которого ионы находятся в ионизационной камере, обычно составляет несколько миллисекунд. Известно, что при атмосферном давлении молекула испытывает $\sim 10^{10}$ столкновений в секунду. Поэтому за время движения иона внутри камеры ионизации он испытывает $\sim 10^{7}$ столкновений, что приводит к изменению состава и концентрации ионов за счет рекомбинации и вторичных реакций с передачей заряда. Определенную сложность при численном моделировании ионного интерфейса представляет также задание начального распределения ионов, вид которого обусловлен различными эффектами поглощения широкополосного жесткого УФ излучения лазерной плазмы буферным газом и присутствующими в нем органическими молекулами.

Ниже мы сначала рассматриваем экспериментальную установку, представляющую собой атмосферный ионный источник с фотоионизацией ионов импульсным излучением лазерной плазмы, затем представляем общую математическую модель эволюции ансамбля ионов под действием газодинамических потоков и электрических полей, создаваемых как внешними электродами, так и коллективным кулоновским взаимодействием между ионами. Модель содержит систему уравнений Навье-Стокса для описания формирования газодинамических потоков в исследуемом объеме, уравнение Пуассона для определения результирующего распределения электрического поля в этом объеме, а также уравнение Лоренца для построения ионных траекторий. Далее мы применяем разработанную математическую модель для оценки ионного тока в упомянутом выше источнике ионов и сравниваем результаты расчетов с экспериментальными данными, полученными при ионизации молекул анилина.

\section{1. Эксперимент}

Измерения проводились на Q-TOF масс-спектрометре с атмосферным вводом, схема которого приведена на рис. 1. Ионизация происходила в герметичной камере из нержавеющей стали 1 , снабженной устройством ввода

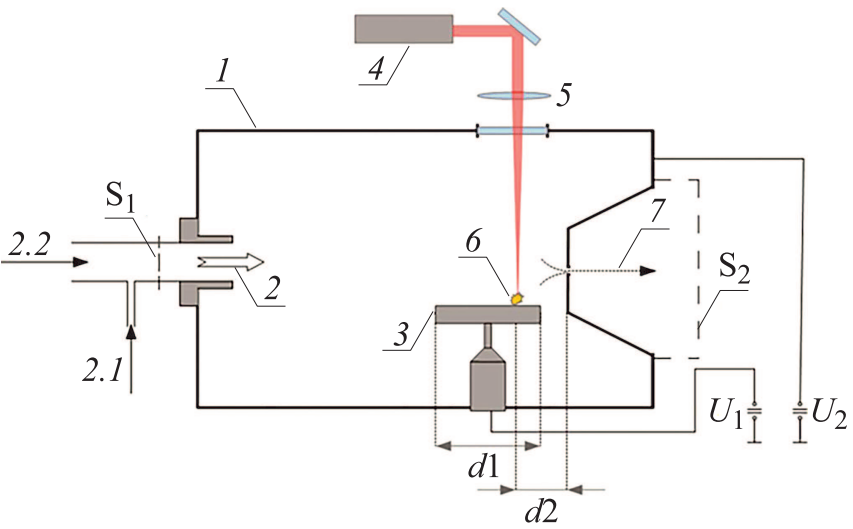

Рис. 1. Схема источника ионов: 1 - камера ионизации; 2 - входной поток буферного газа и исследуемого вещества: 2.1 - поток смеси паров анилина и азота, 2.2 - поток азота; 3 - металлическая мишень; 4 - лазер; 5 - фокусирующая линза; 6 - лазерная плазма на поверхности мишени; 7 поток газа в вакуумную часть масс-спектрометра. Вспомогательные поверхности $\mathbf{S}_{1}$ и $\mathbf{S}_{2}$, обозначенные штриховыми линиями, используются для задания граничных условий при численном решении уравнения Навье-Стокса (см. ниже).

газа 2 и измерителями расхода и давления. Все эксперименты проводились при давлении, близком к атмосферному. Для генерации лазерной плазмы 6, жесткое УФ излучение которой использовалось для ионизации, применялся твердотельный ND: YAG лазер $4(\lambda=1.06 \mu \mathrm{m})$ с длительностью импульса $0.5 \mathrm{~ns}$ и частотой повторений $10 \mathrm{~Hz}$. Лазерный луч попадал в камеру через стеклянное окно и фокусировался на поверхности вращающейся мишени 3 диаметром $d_{1}=10 \mathrm{~mm}$ линзой с фокусным расстоянием $60 \mathrm{~mm}$ в пятно диаметром около $30 \mu \mathrm{m}$. Плотность мощности лазерного излучения на поверхности мишени составляла $w \sim 10^{11} \mathrm{~W} / \mathrm{cm}^{2}$.

Расстояние от места образования плазмы на мишени до плоскости диафрагмы составляло $d_{2}=3 \mathrm{~mm}$. Ионизованные УФ излучением плазмы частицы анализируемого вещества двигались в газовом потоке под воздействием электростатического поля, образованного разностью потенциалов между мишенью и корпусом камеры. Далее ионы через отверстие в диафрагме 7 диаметром $300 \mu \mathrm{m}$ попадали в вакуумную камеру первой ступени откачки масс-спектрометра с давлением 3 Torr.

В качестве исследуемого вещества использовался анилин $\left(\mathrm{C}_{2} \mathrm{H}_{5} \mathrm{NH}_{2}\right)(M=93 \mathrm{a} . \mathrm{m}$. u., давление насыщенных паров $0.6 \mathrm{~mm} \mathrm{Hg})$, а в качестве буферного газа чистый азот $(99.99 \%)$, поток которого в камеру регулировался ротаметром. Пары анилина подавались в камеру в смеси с азотом через специальный капилляр диаметром $100 \mu \mathrm{m}$ и длиной $1 \mathrm{~m}$, соединяющий ампулу, содержащую смесь насыщенного пара анилина с азотом при атмосферном давлении, с камерой ионизации. Поток аналита регулировался за счет изменения перепада давления на капилляре и при перепаде в 30 Torr составлял $5.4 \cdot 10^{14}$ молекул анилина в секунду. 


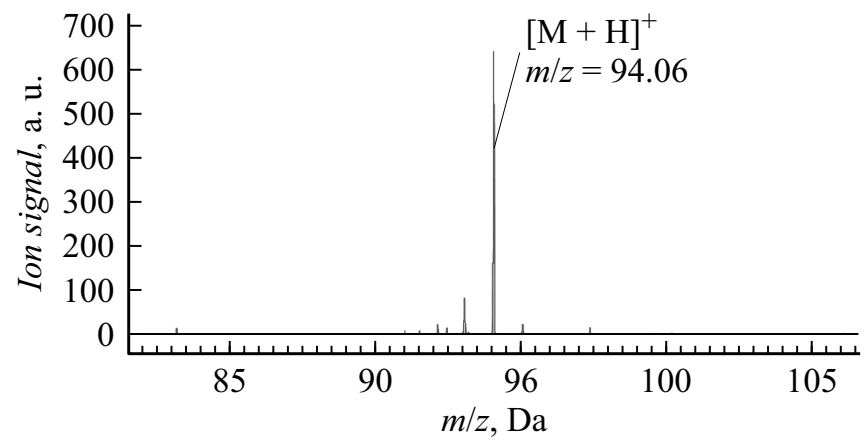

Рис. 2. Ионный сигнал протонированной молекулы анилина, наблюдаемой в масс-спектре.

Анализ временной зависимости интенсивности отдельных линий и непрерывного излучения, наблюдаемых в спектре, показал, что температура плазмы в условиях эксперимента превышала $5 \mathrm{eV}$ в течение первых нескольких наносекунд; УФ излучение такого источника способно ионизовать буферный газ азот. При сечении ионизации порядка $10^{-18} \mathrm{~cm}^{2}$ область сильно ионизованного азота при атмосферном давлении составляет менее $1 \mathrm{~mm}$. Непоглощенное в этом слое УФ излучение горячей плазмы, спектр которого простирается существенно дальше (см. $[13,14])$ длины волны $\lambda \approx 130 \mathrm{~nm}$, соответствующей краю поглощению азота, может распространяться в камере, поскольку концентрация неконтролируемых примесей (в основном паров воды и кислорода) достаточно мала и составляет $\sim 10^{-4}$. Это излучение создает облако ионов воды и молекулярных ионов аналита. В результате вторичных реакций в облаке происходит формирование протонированных ионов аналита, в основном определяющих ионный ток, регистрируемый масс-спектрометром. Основным пиком в масс-спектре (рис. 2) были протонированные молекулы анилина, образованные в результате вторичных реакций с гидроксонием (М - молекула анилина):

$$
M+\mathrm{H}_{3} \mathrm{O}^{+} \rightarrow[\mathrm{M}+\mathrm{H}]^{+}+\mathrm{H}_{2} \mathrm{O} .
$$

В эксперименте измерялась зависимость ионного тока протонированных молекул анилина от разности потенциалов на мишени 3 и на корпусе камеры ионизации 1. Измерения проводились в автоматическом режиме с использованием разработанных в лаборатории средств автоматизированного управления отдельными элементами масс-спектрометра.

\section{2. Постановка задачи численного моделирования}

Численное моделирование динамики ионов в потоке газа под воздействием электрического поля включает в себя численное решение стационарного уравнения Навье-Стокса, уравнения Лапласа для потенциала электростатического поля, создаваемого внешними электродами, и уравнения Лоренца для ионных траекторий с учетом объемного заряда ионного пучка. Поскольку концентрация ионов анилина в азоте мала (соотношение потоков анилина и азота $\sim 10^{-5}$ ), предполагалось, что их присутствие не влияет на динамику течения газа внутри камеры. Поле „зеркального изображения“, обусловленное взаимодействием ионного пучка с проводящей поверхностью электродов, предполагалось пренебрежимо малым. Эти предположения позволили провести моделирование в два этапа. Сначала вычислялось поле газодинамических скоростей и электрическое поле внутри камеры ионизации, а затем моделировалось движение ионов с учетом полученных данных и объемного заряда самого ионного пучка.

Система уравнений Навье-Стокса [15] включает в себя закон сохранения масс, момента импульса и энергии:

$$
\begin{gathered}
\partial \rho / \partial t+\operatorname{div} \rho \mathbf{v}=0 \\
\partial(\rho \mathbf{v}) / \partial t+\nabla \cdot(\rho \mathbf{v v})=-\nabla P+\nabla \cdot(\overline{\bar{\tau}})+\rho \mathbf{g}, \\
\partial(\rho h) / \partial t+\nabla \cdot(\rho h \mathbf{v})=-\nabla \cdot\left[\left(k+k_{t}\right) \nabla T\right],
\end{gathered}
$$

где $\rho-$ плотность газового потока, $\mathbf{v}-$ скорость газового потока, $P$ - давление, $g-$ ускорение свободного падения, $\overline{\bar{\tau}}$ - тензор напряжений,

$$
\overline{\bar{\tau}}=\mu\left[\left(\nabla \mathbf{v}+\nabla \mathbf{v}^{T}-2 / 3 \nabla \cdot \mathbf{v} I\right)\right],
$$

$\mu$ вязкость, I - единичный тензор. Переменная $h$ в уравнении (3) обозначает энтальпию $\left(h=\int_{T_{r e f}}^{T} c_{p} d T, T_{r e f}=298.15 \mathrm{~K}\right), k$ - теплопроводность, $k_{t}$ - теплопроводность при наличии турбулентности $\left(k_{t}=c_{p} \mu_{t} / P r_{t}, P r_{t}\right.$ - число Прандтля, $c_{p}-$ удельная теплоемкость при постоянном давлении).

Численное решение системы уравнений (1)-(3) с граничными условиями для потока $\mathbf{J}$ газа на входной поверхности $S_{1}$ и давления $P$ на поверхности $S_{2}$, расположенной в низковакуумной части масс-спектрометра, и начальной температурой, одинаковой по всей расчетной области

$$
\begin{gathered}
\left.\mathbf{J}\right|_{S_{1}}=\mathbf{J}_{0}, \\
\left.P\right|_{S_{2}}=P_{0}, \\
\left.T\right|_{t=0}=T_{0},
\end{gathered}
$$

осуществлялось методом установления с применением модуля ANSYS CFX пакета программ ANSYS [16]. Потенциал электростатического поля $\varphi$ искался в виде линейной комбинации $\varphi=U_{1} \varphi_{1}+U_{2} \varphi_{2}$,единичных“ функций $\varphi_{1}, \varphi_{2}$, удовлетворяющих уравнению Лапласа $\Delta \varphi=0$ с граничными условиями $\varphi_{1}=1, \varphi_{2}=0$ на поверхности металлической мишени и $\varphi=0, \varphi_{2}=1$ на корпусе камеры ионизации. Численное решение уравнения Лапласа находилось также с помощью программного модуля ANSYS CFX.

Для моделирования движения ионов с учетом поля скоростей буферного газа, статических электрических полей и кулоновского взаимодействия между ионами 
использовалась разработанная авторами программа, написанная на языках C/C ++ в среде Visual Studio 2015 Community. Входными данными для этой программы являются полученные в модуле ANSYS CFX координаты узлов расчетной сетки, сеточные значения давления, компонент вектора скорости газового потока, температуры и компонент вектора напряженности электрического поля. Расчет движения ионов производился многократно для различных значений напряжений между мишенью и корпусом камеры (см. ниже). Для автоматизации работы программы, моделирующей движение ионов, обработки результатов и визуализации использовалась программа GNU Octave.

Моделирование движения ионов осуществлялось от момента их зарождения при воздействии короткого импульса УФ излучения плазмы. Как указывалось выше, время существования жесткого непрерывного УФ излучения плазмы составляет несколько десятков наносекунд, при этом ионы достигают диафрагмы в среднем за несколько миллисекунд. Поэтому при моделировании предполагалось, что облако первичных ионов (как положительных, так и отрицательных), создаваемых излучением плазмы за счет фотоионизации, „рождается“ мгновенно в начальный момент времени. В рамках модели плотность образованных ионов предполагалась зависимой от плотности нейтральных частиц и потока УФ излучения. Плотность нейтральных частиц в момент ионизации полагалась равномерной по всему объему и определялась из соотношения потоков исследуемого вещества и буферного газа. Предполагалось также, что интенсивность излучения плазмы падает как $\left(1 / r^{2}\right) \exp (-a r)$, где функция $1 / r^{2}$ соответствует плотности излучения точечного источника, а фактор $\exp (-a r)$ отвечает за поглощение излучения средой; при этом концентрация протонированных молекул анилина была пропорциональна плотности УФ-излучения.

Ионные траектории определялись в результате численного решения уравнения Лоренца для индивидуальной частицы

$$
m \mathbf{a}=q \mathbf{E}+\mathbf{F}_{g}+\mathbf{F}_{c},
$$

где $m$ - масса иона, а - ускорение иона, $q$ - заряд иона, $\mathbf{E}$ - напряженность электростатического поля, $\mathbf{F}_{g}$ - газодинамическая составляющая силы, действующей на ион, $\mathbf{F}_{c}-$ кулоновская сила, действующая со стороны ансамбля ионов на индивидуальную частицу. Сила $\mathbf{F}_{g}$, обусловленная столкновениями иона с молекулами буферного газа, определялась как [17]

$$
\mathbf{F}_{g}=q\left(\mathbf{V}_{g}-\mathbf{V}\right) / k,
$$

где $\mathbf{V}_{g}$ и $\mathbf{V}-$ скорости газового потока и иона соответственно, $k$ - коэффициент подвижности, зависящий от давления $P$ и температуры $T$ [18]:

$$
k(P, T)=k_{0} P_{0} / P \sqrt{T / T_{0}} .
$$

Здесь $k_{0}, P_{0}, T_{0}$ - коэффициент подвижности, давление и температура при нормальных условиях [19]. Для легких ионов уравнение движения приобретает вид [20]:

$$
\mathbf{V}=\mathbf{V}_{g}+k \mathbf{E}+k / q \mathbf{F}_{c} .
$$

Для моделирования ионных ансамблей с большим $\left(\sim 10^{6}\right)$ количеством частиц нами использовался подход, основанный на идеологии метода „крупных частиц“ [21]. Вместо большого числа частиц с массой $m$ и зарядом $z$ рассматривались „крупные частицы“ с массой $M=b m$ и зарядом $Z=b z$, где $b-$ коэффициент умножения. $\mathrm{B}$ настоящей работе $b$ полагался равным 1000. Такой подход позволил существенно увеличить скорость счета. При этом можно считать, что движение „крупных частиц“определяется теми же законами, что и движение частиц массы $m$. Действительно, из формул (5) и (6) видно, что ускорение, приобретаемое частицей за счет действия электрического и газодинамического полей, пропорционально отношению заряда частицы к ее массе. А поскольку масса и заряд „крупных частиц“ пропорциональны массе и заряду исходной частицы (с одним и тем же коэффициентом пропорциональности), действие электрического и газодинамического полей на „крупную частицу“ оказывается таким же, как и на исходную.

Количество таких „крупных частиц“ в наших численных экспериментах составляло 1000. Учет кулоновского взаимодействия между „квазичастицами“ осуществлялся на основе метода Барнса-Хата [22].

\section{3. Обсуждение результатов}

На рис. 3 показано сечение вычислительной сетки, сгенерированной программой ANSYS для решения газодинамической и электростатической задач. Хорошо видно, что сетка весьма неоднородна и существенно сгущается по мере приближения к диафрагме, что отражает сильную разномасштабность рассматриваемой задачи. Рис. 4 иллюстрирует сходимость метода установления при численном решении уравнений (1)-(3). Показанная на этом рисунке максимальная (по узлам вычислительной сетки) относительная невязка осевой компоненты векторного уравнения Навье-Стокса (2) за $5 \cdot 10^{4}$ итераций метода установления уменьшилась на 4 порядка от величины $\sim 1$ до величины $\sim 10^{-4}$. Невязка по другим компонентам уравнения Навье-Стокса вела себя аналогичным образом. Одновременно невязка уравнения непрерывности (1) за то же количество итераций уменьшилась более чем на 5 порядков.

На рис. 5 показано распределение модуля вектора скорости газового потока в сечении, проходящем через центр диафрагмы перпендикулярно плоскости мишени. Скорость потока вблизи точки возникновения лазерной плазмы на мишени составляет $\sim 0.1 \mathrm{~m} / \mathrm{s}$ и, возрастая до $\sim 200 \mathrm{~m} / \mathrm{s}$ в плоскости диафрагмы, на выходе из диафрагмы превышает локальную скорость звука.

На рис. 6 показано распределение электрического поля в камере ионного источника. Эквипотенциали соответствуют распределению электростатического потенциала в плоскости сечения, проходящей через центр 


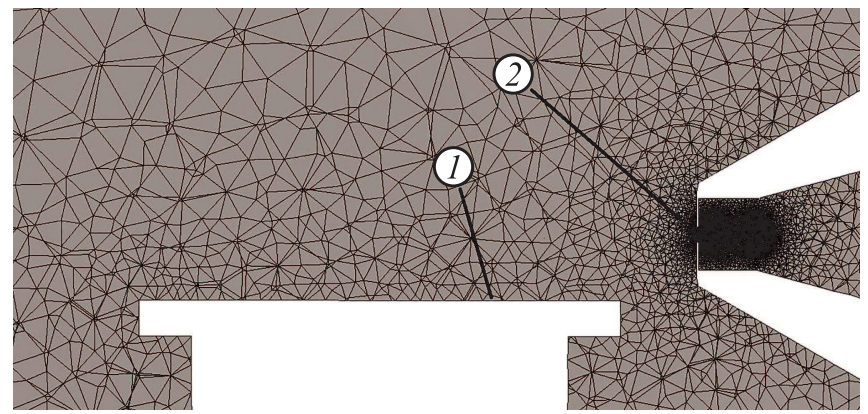

Рис. 3. Вычислительная сетка для решения газодинамической и электростатической задач: 1 - поверхность мишени; $2-$ диафрагма.

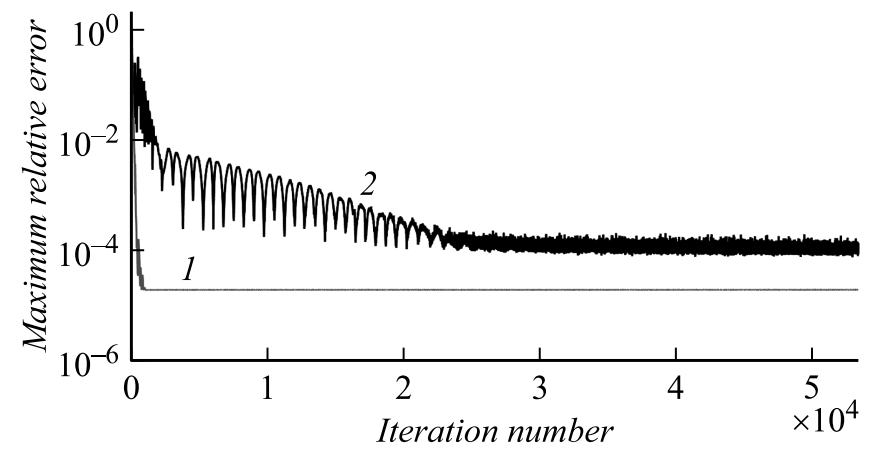

Рис. 4. Зависимость максимальной относительной невязки в уравнении непрерывности (кривая 1) и в уравнении Навье-Стокса (кривая 2).

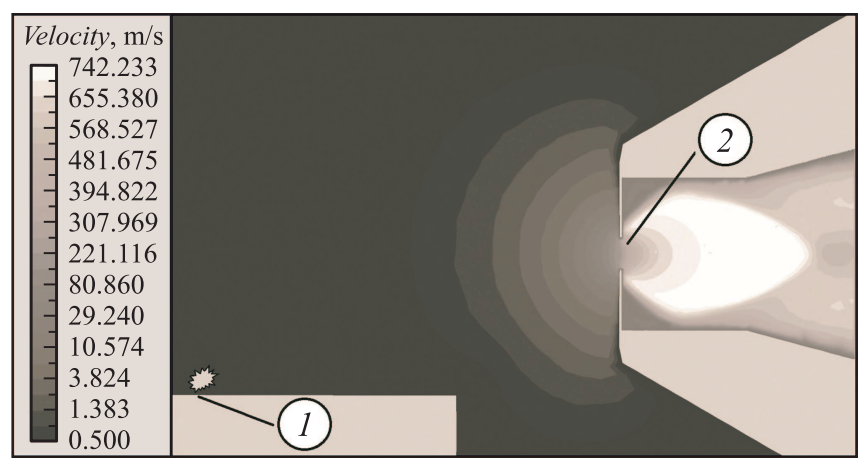

Рис. 5. Распределение модуля скорости газового потока в плоскости сечения, проходящего через центр диафрагмы перпендикулярно плоскости мишени: 1 - плазма на поверхности мишени, 2 - диафрагма.

диафрагмы перпендикулярно плоскости мишени при разности потенциалов между мишенью $\left(U_{T}\right)$ и корпусом $\left(U_{C}\right)$ камеры ионизации $\Delta U=10 \mathrm{~V}$.

Были проведены две серии вычислительных экспериментов, по-разному учитывающих время жизни ионов аналита (эффект рекомбинации). В первой серии предполагалось, что время жизни индивидуального иона при его движении к диафрагме не ограничено. Начальное распределение ионов показано на рис. 7.
Во второй серии вычислительных экспериментов эффект рекомбинации учитывался путем ограничения времени жизни ионов и соответственно области „достижимости“ диафрагмы ионами на основе допущения, что ионы, лежащие вне некоторой области, показанной на рис. 8, не достигают диафрагмы, и поэтому не дают вклада в результирующий ионный ток.

На рис. 9 приведена экспериментальная зависимость тока положительных ионов от напряжения между мишенью и корпусом камеры ионизации масс-спектрометра

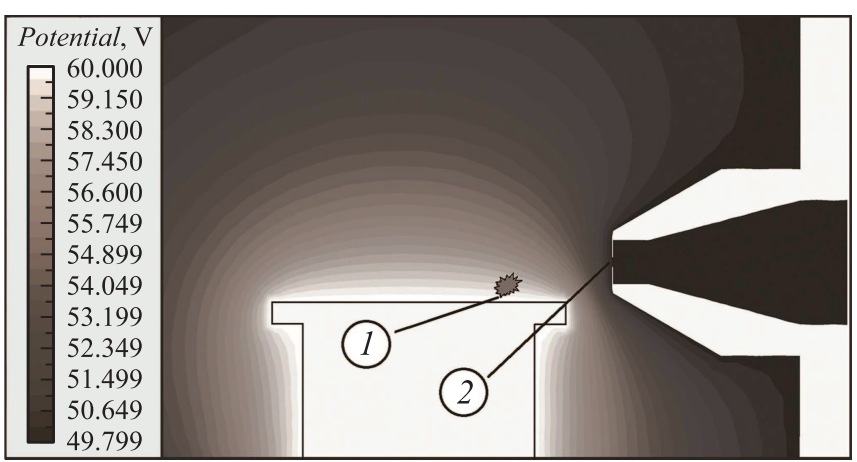

Рис. 6. Распределение потенциала электрического поля в плокости сечения, проходящей через центр диафрагмы перпендикулярно плоскости мишени при разности напряжений между мишенью и корпусом камеры ионизации $\Delta U=10 \mathrm{~V}$ : 1 - плазма на поверхности мишени, 2 - диафрагма.

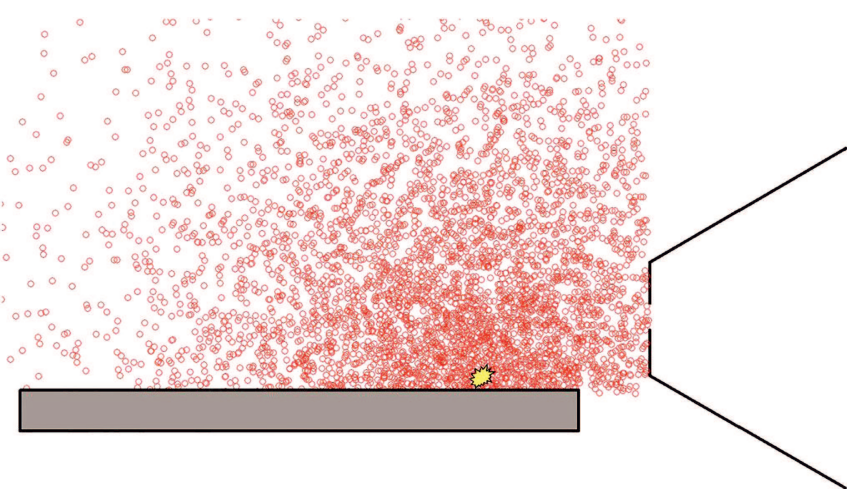

Рис. 7. Начальное распределение ионов. Желтым (в on-line версии) отмечено место образования плазмы на поверхности мишени.

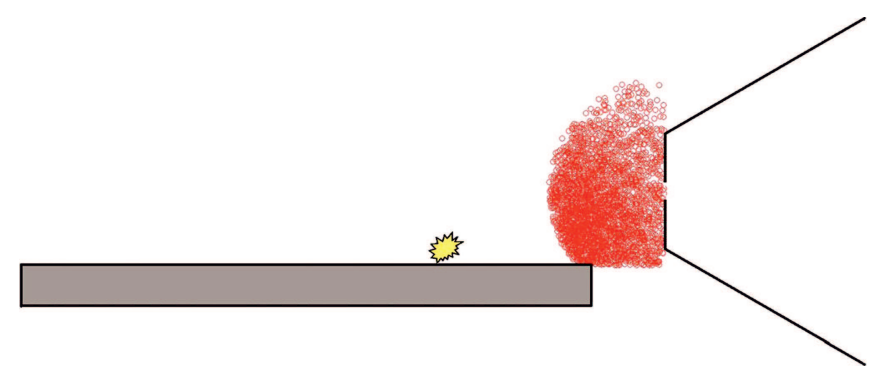

Рис. 8. Начальное распределение ионов в предположении конечного времени жизни ионов из-за процессов рекомбинации. 


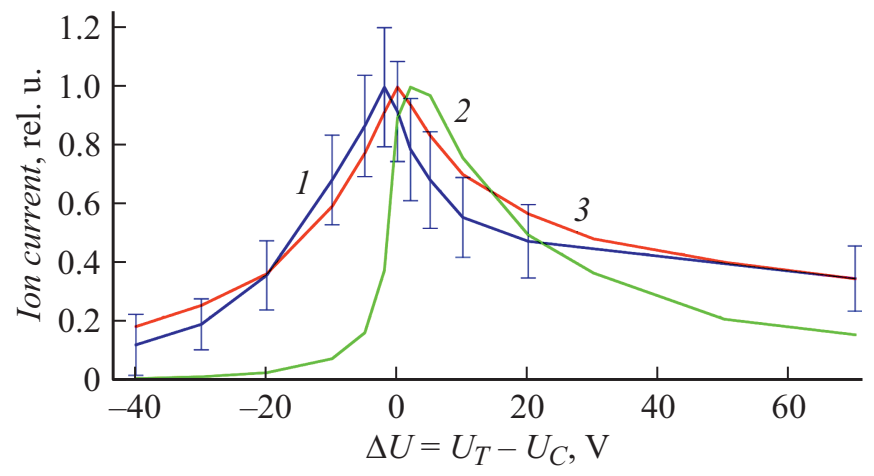

Рис. 9. Зависимость ионного тока от разности потенциалов на мишени и на корпусе: 1 - экспериментальная кривая, 2 результат моделирования с начальным распределением, показанным на рис. 7, 3 - результат моделирования с начальным распределением, показанным на рис. 8.

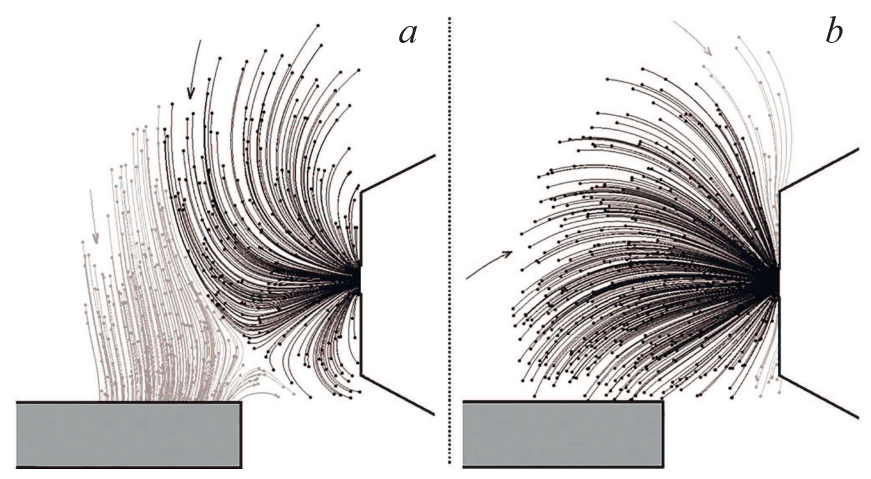

Рис. 10. Траектории движения ионов внутри камеры ионизации (вид сбоку): $a-$ при $U_{T}-U_{C}=-10 \mathrm{~V} ; b-$ при $U_{T}-U_{C}=10 \mathrm{~V}$. Траектории темного цвета проходят сквозь диафрагму, светлые - заканчиваются на стенках диафрагмы.

(кривая 1). Такая зависимость оказалась характерной для ионов разных масс с незначительными изменениями формы кривой. Появление „хвоста“ ионного тока в области достаточно сильных тормозящих (для положительных ионов) электрических полей, по-видимому, обусловлено эффектом рекомбинации, который в данном случае проявляется в том, что в коллектор массспектрометра попадают только ионы, „рожденные“ в сильном газовом потоке вблизи отверстия диафрагмы.

Как видно из рис. 9, кривая 2, полученная в первой серии расчетов, и экспериментальная зависимость ионного тока через диафрагму от разности потенциалов между мишенью и корпусом камеры ионизации довольно сильно отличаются. В то же время, кривая 3, полученная во второй серии расчетов с учетом эффекта рекомбинации, значительно лучшее соответствует эксперименту (рис. 9).

В области $U_{T}-U_{C}<0$ ионный сигнал падает изза того, что электрическое поле уносит заряженные частицы от диафрагмы. В качестве примера на рис. 10, $a$ представлены траектории движения ионов при разности потенциалов между мишенью и камерой $-10 \mathrm{~V}$. Для удобства визуализации количество траекторий уменьшено. Ионы, стартующие из области, близкой к мишени, где электрическая составляющая преобладает над газодинамической, не попадают в вакуумную часть массспектрометра. С дальнейшим уменьшением потенциала мишени по отношению к потенциалу камеры через диафрагму проходят только те ионы, которые образовались вблизи нее, и при скорости газового потока, достигающей в окрестности диафрагмы 40-200 m/s, эти ионы вместе с мощным газовым потоком „всасываются“ в диафрагму.

Обратная ситуация возникает в случае, когда потенциал на мишени больше потенциала на камере ионизации $\left(U_{T}-U_{C}>0\right)$. В этом случае ионы, уносимые газовым потоком, получают дополнительное ускорение под действием электрического поля, направленного от мишени к стенкам камеры ионизации. Из области, близкой к торцу входной части масс-анализатора, где расположена диафрагма, в вакуумную часть попадают ионы, расположенные близко к оси диафрагмы. В противном случае они „теряются“ при столкновении с торцевой плоскостью. На рис. 10, $b$ представлены траектории ионов при разнице напряжений $U_{T}-U_{C}=10 \mathrm{~V}$. Отметим, что при дальнейшем увеличении напряжения между электродами ионы, находящиеся на достаточном удалении от диафрагмы, погибают на стенках камеры. В области, удаленной от диафрагмы, скорость газового потока мала, поэтому движение ионов в этой области обусловлено только электрической составляющей; при этом вектор напряженности поля направлен от мишени к стенкам ионизационной камеры.

\section{Заключение}

Чрезвычайное разнообразие и сложность физических процессов ионизации вещества УФ излучением лазерной плазмы делает задачу численного моделирования этих процессов весьма сложной. В значительной степени трудности моделирования обусловлены также и тем, что экспериментальные данные о природе излучения лазерной плазмы, поглощения такого излучения средой и различных цепочек химических реакций, приводящих к ионизации аналита, являются недостаточно полными. То же самое относится и к описанию процессов рекомбинации и переноса заряда при движении ионного ансамбля в газообразной среде.

Нами рассмотрены результаты численного моделирования импульсного источника ионов атмосферного давления с ионизацией исследуемого вещества УФ излучением лазерной плазмы. Расчеты проводились на основе разработанной математической модели, описывающей эволюцию ансамбля ионов под действием газодинамических и электрических полей с учетом эффектов кулоновского взаимодействия между ионами. Модель ориентирована на расчет характеристик атмосферных интерфейсов, используемых в масс-спектрометрии, и 
включает в себя систему уравнений Навье-Стокса для описания формирования газодинамических течений в исследуемом объеме, уравнение Пуассона для определения электрического поля в этом объеме и уравнение Лоренца для построения траекторий ионов от места их рождения до коллектора. Продемонстрирована сходимость релаксационного метода к численному решению основных уравнений модели. Численные эксперименты показали, что конечность времени жизни ионов вследствие эффекта рекомбинации радикально влияет на эффективность сбора ионов. Учет этого фактора при численном моделировании ионного источника позволил получить хорошее согласие расчетных и экспериментальных зависимостей ионного тока на коллекторе от приложенного напряжения. Получен важный для практической реализации метода результат, определяющий размер области дрейфа ионов, который используется в конструировании ионных источников, обеспечивающих высокую чувствительность детектирования органических соединений при использовании масс-спектрометров с ионизацией при атмосферном давлении.

Все это свидетельствует о том, что уже на данном этапе представленная модель может быть использована для разработки и оптимизации ионных интерфейсов c импульсной ионизацией при атмосферном давлении. В целом полученные результаты можно рассматривать как определенный шаг в направлении создания более сложных и полных моделей для количественного описания физических процессов, происходящих в атмосферных ионных интерфейсах, применяемых в массспектрометрии.

\section{Конфликт интересов}

Авторы заявляют, что у них нет конфликта интересов.

\section{Список литературы}

[1] C.M. Whitehouse, R.N. Dreyer, M. Yamashita, J.B. Fenn. Anal. Chem., 57 (3), 675 (1985).

[2] R. Javanshad, A. Venter. Anal. Methods, 9 (34), 4896 (2017).

[3] R.B. Cody, J.A. Laramee, H.D. Durst. Anal. Chem., 77 (8), 2297 (2005).

[4] M. Smoluch, P. Mielczarek, J. Silberring. Mass Spectrom. Rev., 35 (1), 22 (2016).

[5] D.B. Robb, T.R. Covey, A.P. Bruins. Anal. Chem., 72 (15), 3653 (2000).

[6] А.В. Пенто, С.М. Никифоров, Я.О. Симановский, А.А. Гречников, С.С. Алимпиев. Квант. электрон., 43 (1), 55 (2013). [A.V. Pento, S.M. Nikiforov, Y.O. Simanovsky, A.A. Grechnikov, S.S. Alimpiev. Quant. Electron., 43 (1), 55 (2013).]

[7] M. Constapel, M. Schellentrager, O. Schmitz, S. Gab, K. Brockmann, R. Giese, T. Benter. Rapid Commun. Mass Spectrom., 19 (3), 326 (2005).

[8] С.И. Анисимов, Б.С. Лукьянчук. УФН, 172 (2), 301 (2002). [S.I. Anisimov, B.S. Luk'yanchuk. Physics-Uspekhi, 45 (3), 293 (2002).]
[9] E.G. Gamaly, A.V. Rode, V.T. Tikhonchuk, B. Luther-Davies. Phys. Plasmas, 9 (3), 949 (2002).

[10] A.H. Lutey. J. Appl. Phys., 114, 083108 (2013).

[11] A.H. Hamad. High energy and short pulse lasers. 2016, Chapter 12. P. 305.

[12] S.S. Harilal, B. O’Shay, M.S. Tillack, Y. Tao, R. Paguio, A. Nikroo, C.A. Back. J. Phys. D: Appl. Phys., 39 (3), 484 (2006).

[13] H.C. Liu, X.L. Mao, J.H. Yoo, R.E. Russo. Spectrochim. Acta Part B, 54, 1607 (1999).

[14] N. Farid, S.S. Harilal, H. Ding, A. Hassanien. J. Appl. Phys., 115, 0331071 (2014).

[15] Ansys CFX-Solver Theory Guide (Ansys, Inc, 2018, p. 21)

[16] Ansys CFX, Canonsburg, PA, USA, http://www.ansys.com

[17] M.G. Skoblin, A.V. Chudinov, I.V. Sulimenkov, V.S. Brusov, A.A. Makarov, E.R. Wouters, V.I. Kozlovskiy. Eur. J. Mass Spectrom., 23 (4), 187 (2017).

[18] Л.Д. Ландау, Е.М. Лифшиц. Физическая кинетика. (Физматлит, М., 2002)

[19] И.А. Буряков. ЖТФ, 74 (8), 15 (2004).

[20] А.Д. Андреева, А.А. Елистратов, Л.Н. Галль. Научное приборостроение, 16 (2), 73 (2006).

[21] О.М. Белоцерковский, Ю.М. Давыдов. Метод крупных частии, в газовой динамике. (Наука, М., 1982)

[22] J. Barnes, P. Hut. Nature, 324 (4), 446 (1986). 